\title{
An Unusual Migration of A Stent: A Case Report
}

Fikadu G. Tekleyes MD ${ }^{1}$

Steven Craig RTR ${ }^{2}$

Ellen A. Thompson $\mathrm{MD}^{1}$

Mark A. Studeny MD ${ }^{1}$

Melissa D. Lester DO ${ }^{1}$

\section{Author Affiliations:}

1. Marshall University, WV

2. Cabell Huntington Hospital, WV

The authors have no financial disclosures to declare and no conflicts of interest to report.

\section{Corresponding Author:}

Fikadu G. Tekleyes MD

Department of Cardiology

Marshall University Joan C Edwards School of Medicine

Huntington, WV

Email: fikadumd@aol.com 


\section{Abstract:}

Stent dislodgment and embolization is a rare complication of coronary stenting. There are reports of intra-coronary stent entrapment, stripping, and dislodgement during percutaneous coronary interventions causing potentially life threatening complications, including intra-coronary or systemic embolization. Reports of dislodgment and migration of previously placed drug eluting intra-coronary stent several months after deployment are very limited in the literature. We describe a drug eluting stent dislodgment and migration in an 88 year-old male 10 months after its deployment.

\section{Introduction:}

Stent dislodgement and embolization is a rare complication of percutaneous coronary intervention. There are reports of intra-coronary stent entrapment, stripping and dislodgement during percutaneous coronary interventions. These can cause potentially life-threatening complications to the patient, including intra-coronary or systemic embolization and create a stressful experience to the operators. ${ }^{1-5}$ There are case reports of dislodgment and peripheral embolization of a sirolimus-eluting stent from the ostium of a left main coronary artery. ${ }^{6,7}$ There are case reports reflecting potential for compression, distortion and dislodgement of large endovascular stents in some situations, like CPR. ${ }^{8}$ However, reports of dislodgement and migration of previously placed drug eluting intra-coronary stent several months after deployment are limited. We describe an unusual case of a drug eluting coronary stent displacement 10 months after its deployment.

\section{Case report:}

After a roll over tractor accident, an 84 year-old male was admitted to the surgical intensive care unit with a hip fracture, blunt chest wall injury, and possible pulmonary contusion. He had coronary artery disease and a history of coronary bypass 15 years prior. He had percutaneous intervention to an $80 \%$ stenosis of a saphenous vein graft (SVG) to a right posterior descending artery (PDA) using a 3.0mm x $15 \mathrm{~mm}$ Resolute Integrity drug-eluting stent 10 months prior to his current presentation. He was very active and was taking a beta-blocker, angiotensin receptor blocker, aspirin, Clopidogrel, and a statin. Admission record reflects that aspirin and Clopidogrel had been held since the day of admission for surgical interventions.

On the $3^{\text {rd }}$ hospital day, an internal fixation of his fractured hip was performed. Post procedure, the patient was extubated successfully but was re-intubated due to hypoxemia and tachycardia. The evening of his hip surgery a nursing staff noted ST segment changes on the telemetry monitor. A 12 lead EKG was obtained and showed a dynamic changes in the inferior wall indicating ST elevation in the inferior leads with reciprocal changes in the high lateral leads (as shown on Fig. 1 and 2). A stat cardiology consult was called. 


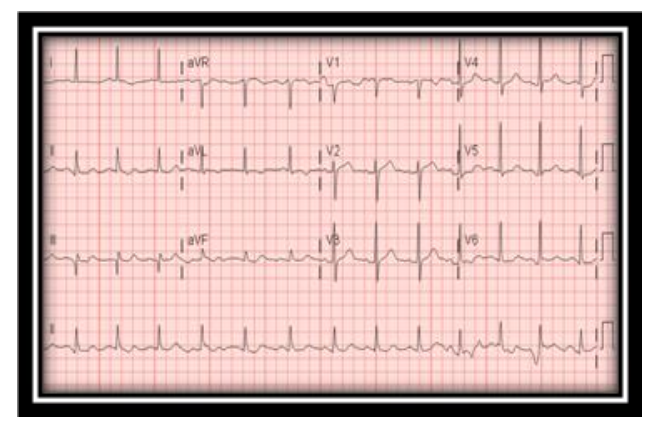

Fig 1. EKG 1. Pre-intervention

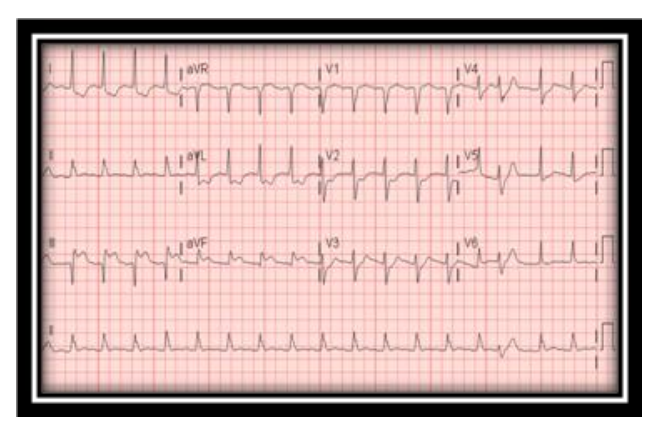

Fig 2. EKG 2. Pre-intervention

\section{Decision Making:}

The decision was made to proceed with emergent left heart catheterization and intervention if indicated. Selective angiogram of the SVG to PDA graft showed a thrombus with a near total occlusion of the previously placed stent ( $3.0 \mathrm{~mm}$ x $15 \mathrm{~mm}$ Resolute Integrity drug-eluting stent) in the distal graft, with very faint antegrade coronary flow beyond the occlusion (TIMI I flow) as shown in Fig. 4.

Multiple thrombus aspirations were performed using export aspiration catheter. This was followed by a balloon angioplasty using $2.5 \mathrm{~mm} \times 20 \mathrm{~mm}$ and $3.0 \mathrm{~mm} \times 20 \mathrm{~mm}$ Emerge over the wire balloons. This resulted in a marked improvement in the thrombus burden and improved flow across the stenotic lesion. Then a $3.0 \mathrm{~mm}$ x $20 \mathrm{~mm}$ resolute Integrity stent was passed across the lesion, and angiographic images obtained to assess the optimal location for deployment.

However, on subsequent images it was noted that there was still a significant residual thrombus at the level of the old stent and the stent balloon was pulled back and withdrawn, prior to deployment, with a plan to attempt further thrombus aspiration. Angiographic views obtained following the withdrawal of the stent balloon showed the old stent was dislocated to almost the mid aspect of the saphenous vein graft and was now causing flow limitation more proximally. Multiple views were obtained to confirm this finding, as this was an unanticipated and unusual complication. The export aspiration catheter was used to reattempt aspiration of the residual thrombus. Then the stenosis at the level of the migrated stent and the distal lesion were treated with balloon angioplasty using $4.0 \mathrm{~mm} \times 20 \mathrm{~mm}$ Trek over-the-wire balloon followed by deployment of two $4.0 \mathrm{~mm}$ x 18mm Xience drug-eluting stents. Subsequent angiographic images showed achievement of a TIMI III flow across the stented lesions and distally (Fig. 5). The procedure was then concluded and the patient was transferred to catheterization laboratory holding for further observation. EKG obtained at the conclusion of the procedure showed resolution of the ST segment elevation and the patient did well on subsequent follow up periods. 

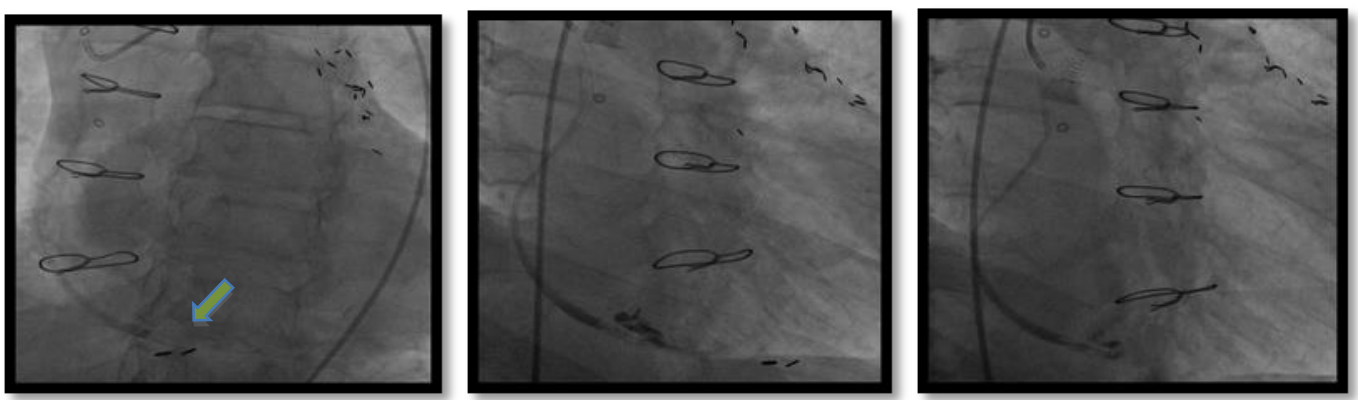

Fig 3. SVG to PDA graft, Pre-intervention
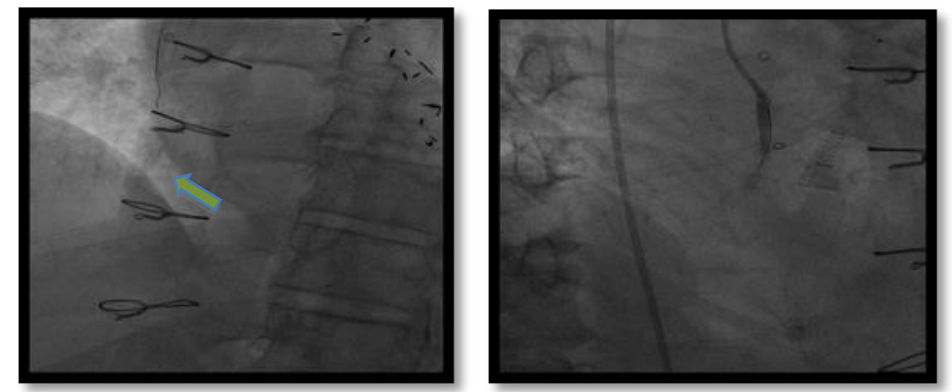

Fig 4. SVG to PDA graft, Post stent migration.
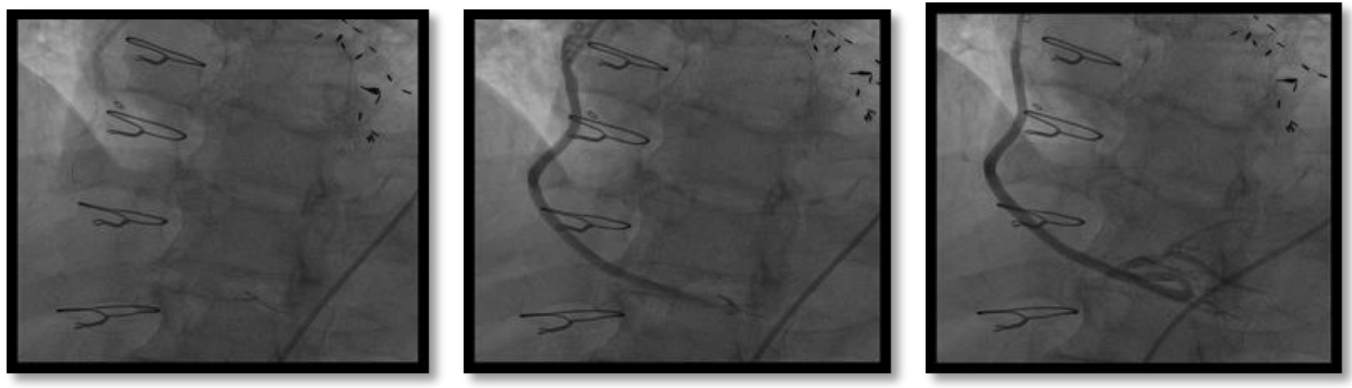

Fig 5. SVG to PDA graft, post stent migration and post-intervention

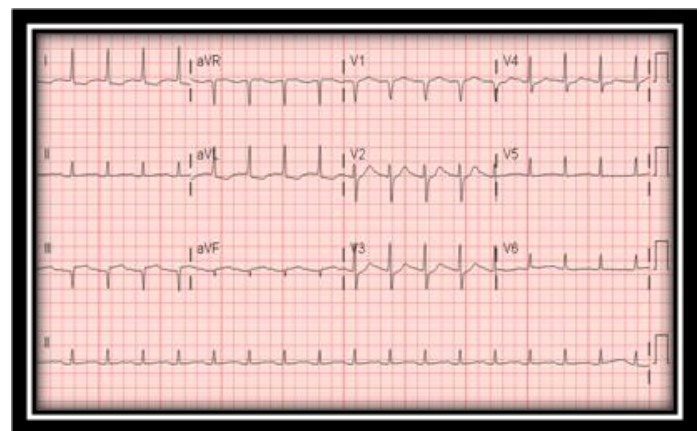

Fig 6. EKG 3: Post-intervention 


\section{Conclusion:}

Coronary stent dislodgment is very rare but critical complication of percutaneous coronary intervention. The incidence varies between $0.32-8.3 \% .{ }^{1-3} \mathrm{~A}$ stent can be dislodged from the stent-balloon assembly during pull back, prior to deployment, of the stent balloon into the guide catheter. Interventions to extremely angulated and severely calcified coronary lesions are more prone to this complication. ${ }^{3,5,9}$ It also can occur during an attempt to intervene on inadequately pre-dilated lesions ${ }^{3}$ or with interference from previously deployed stents. Coronary stent displacement is also reported with use of intracoronary vasodilator therapy during percutaneous coronary intervention. ${ }^{10}$

Although the incidence is decreasing with the development of improved devices and techniques, with the use of pre-mounted stents, we continue to see reports of stent dislodgment, migration and peripheral embolization during the time of percutaneous coronary interventions. ${ }^{1-3}$

However, to our knowledge this is the first report of displacement of a drug eluting stent several months after its deployment. Though the reason behind this dislocation is unclear, it is suspected that it is a result of the entrapment of the new stent balloon in the struts of the old stent, which likely was under-sized and poorly embedded into the vessel wall, leading to this unusual very late dislocation. 


\section{References:}

1. Brilakis ES, Best PJ, Elesber AA, Barsness GW, Lennon RJ, Holmes DR, Jr., et al. Incidence, retrieval methods, and outcomes of stent loss during percutaneous coronary intervention: a large single-center experience. Catheter Cardiovasc Interv. 2005;66(3):333-40.

2. Eggebrecht H, Haude M, von Birgelen C, Oldenburg O, Baumgart D, Herrmann J, et al. Nonsurgical retrieval of embolized coronary stents. Catheter Cardiovasc Interv. 2000;51(4):432-40.

3. Colkesen AY, Baltali M, Acil T, Tekin G, Tekin A, Erol T, et al. Coronary and systemic stent embolization during percutaneous coronary interventions: a single center experience. Int Heart J. 2007;48(2):129-36.

4. Aydin M, Sayin MR. Successful coronary stent retrieval from the saphenous vein graft to right coronary artery. Case Rep Med. 2009;2009:718685.

5. Nikolsky E, Gruberg L, Pechersky S, Kapeliovich M, Grenadier E, Amikam S, et al. Stent deployment failure: reasons, implications, and short- and long-term outcomes. Catheter Cardiovasc Interv. 2003;59(3):324-8.

6. Kubota T, Ishikawa T, Miyamoto T, Mutoh M. Asymptomatic migration of a sirolimus-eluting stent into the aorta. Intern Med. 2010;49(18):2021-2.

7. Won KB, Kim BK, Ko YG, Hong MK, Jang Y, Shim WH. Migration of a sirolimus-eluting stent from the ostium of the left main coronary artery to the right deep femoral artery. Korean J Intern Med. 2013;28(1):116-9.

8. Haas NA, Happel CM, Jategaonkar S, Moysich A, Hanslik A, Kececioglu D, et al. Compression, distortion and dislodgement of large caliber stents in congenital heart defects caused by cardiopulmonary resuscitation: a case series and review of the literature. Clin Res Cardiol. 2014;103(9):719-25.

9. Laarman G, Muthusamy TS, Swart H, Westendorp I, Kiemeneij F, Slagboom T, et al. Direct coronary stent implantation: safety, feasibility, and predictors of success of the strategy of direct coronary stent implantation. Catheter Cardiovasc Interv. 2001;52(4):443-8.

10. Celik M, Cagdas Yuksel U, Gokoglan Y. Migration of fully deployed stent after intracoronary glyceryl trinitrate administration: An unusual percutaneous coronary intervention complication. Pak J Med Sci. 2013;29(3):863-5. 